
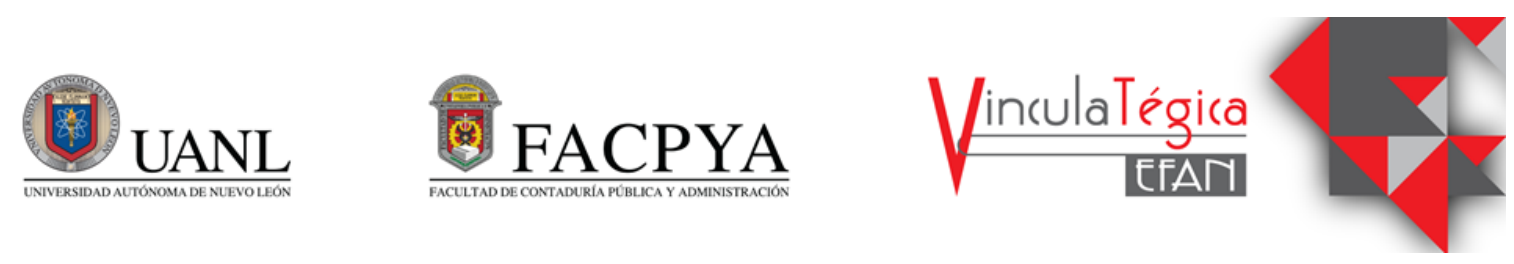

\title{
Relación entre factores de regulación externa y motivación en alumnos de posgrado de Universidad Autónoma del Noreste campus Monclova durante emergencia sanitaria por SARS-CoV2
}

\author{
Karina Lizeth Fabela Godina ${ }^{1}$ y Oscar Mario Farias Montemayor ${ }^{2}$ \\ ${ }^{1}$ Universidad Autónoma del Noreste,dra.karinafabela@gmail.com, Privada Miguel Blanco, Zona Centro, CP \\ 25700, Monclova Coahuila, 8008228263 \\ ${ }^{2}$ Universidad Autónoma del Noreste,ofarias002@hotmail.com, Privada Miguel Blanco, Zona Centro, CP \\ 25700, Monclova Coahuila, 8008228263
}

Información del artículo revisado por pares

Fecha de aceptación: junio-2021

Fecha de publicación en línea: diciembre-2021

DOI: https://doi.org/10.29105/vtga7.2-53

\section{Resumen}

La contingencia sanitaria causada por covid19 ha afectado sistemas gubernamentales, económicos, de salud y educativos a lo largo del mundo. En el sistema educativo ha obligado a las instituciones a migrar súbitamente de modelos presenciales a modelos de educación a distancia o virtuales.

En menor escala durante esta pandemia las personas se han visto impactadas con problemas de salud, emocionales, económicos y laborales. Los alumnos de maestrías de salud ocupacional y terapia familiar de la Universidad Autónoma del Noreste campus Monclova no están ajenos a estos problemas que pueden llevarlos a desmotivación académica y en casos graves deserción del estudiante, por lo que esta investigación tiene por objetivo verificar estadísticamente la existencia de relación entre la motivación por adquirir nuevos conocimientos y los factores de regulación externa del alumno de maestrías en salud ocupacional y terapia familiar de la Universidad Autónoma del Noreste campus Monclova durante emergencia sanitaria por SARS-CoV2. La investigación se llevó

\begin{abstract}
The health contingency caused by covid-19 has affected government, economic, health and educational systems throughout the world. In the educational system, it has forced institutions to suddenly migrate from face-to-face models to distance or virtual education models.

On a smaller scale, during this pandemic, people have been impacted with health, emotional, economic and work problems. The students of master's degrees in occupational health and family therapy at the Universidad Autónoma del Noreste campus Monclova are not exempt from these problems that can lead to academic demotivation and in serious cases of student desertion, so this research aims to statistically verify the existence of a relationship between the motivation to acquire new knowledge and the external regulation factors of the student of master's degrees in occupational health and family therapy of the Universidad Autónoma del Noreste campus Monclova during a health emergency due to SARS-CoV2. The research was carried out from February to March 2021 at the Universidad Autónoma del Noreste campus Monclova taking as a sample 35 graduate students
\end{abstract}


durante febrero a marzo de 2021 en la Universidad Autónoma del Noreste campus Monclova teniendo como muestra 35 alumnos de posgrado de maestrías de Salud Ocupacional y Terapia Familiar. Para recabar información se aplicó el instrumento "Escala de Motivación Educativa". Al realizar prueba de correlación de Spearman en variables se obtiene que "no existe relación significativa entre los factores de regulación externa y la motivación para adquirir conocimientos por parte de los alumnos de las maestrías de salud ocupacional y terapia familiar de la Universidad Autónoma del Noreste campus Monclova durante emergencia sanitaria por SARS-CoV2.

Palabras clave: Covid-19, educación a distancia, y motivación.

\section{INTRODUCCIÓN}

Durante el mes de diciembre se presentó "un grupo de 27 casos de neumonía de etiología desconocida, detectados en el municipio de Wuhan en la provincia de Hubei, China" (Hernández et al., 2020, p. 59), lo cual fue dado a conocer mediante un comunicado de prensa por parte de la Organización Mundial de la Salud (OMS) en su página de internet donde declaró que "la Comisión Municipal de Salud de Wuhan (provincia de Hubei, China) notifica un conglomerado de casos de neumonía en la ciudad. Posteriormente se determina que están causados por un nuevo coronavirus" (Paniagua, 2020, p. 155), y a partir del "11 de marzo de 2021 la Organización Mundial de la Salud declara la pandemia mundial" (Muñiz y Corduneanu, 2020, p. 45). El término pandemia proviene de los vocablos formada por "pan (todo) y demos (pueblo). Designa una enfermedad que from Master's degrees in Occupational Health and Family Therapy. To collect information, the instrument "Educational Motivation Scale" was applied. When performing the Spearman correlation test on variables, it is obtained that "there is no significant relationship between external regulation factors and the motivation to acquire knowledge on the part of the students of the master's degrees in occupational health and family therapy at the Universidad Autónoma del Noreste campus Moncova during a health emergency due to SARS-CoV2.

Keywords: Covid-19, distance education and motivation.

JEL: I21, I23.

afecta a todo un pueblo" (Galli, 2020), por lo que "el término "pandemia" podría aplicarse a cualquier epidemia que logre extenderse ampliamente" (Álvarez et al., 2020, p. 24) así como a aquella "enfermedad epidémica que se extiende a muchos países o que ataca a casi todos los individuos de una localidad o región” (Balleti, 2020, 190).

Esta pandemia es causada por "la enfermedad por COVID-19 o novel coronavirus, es una pandemia global de enfermedad respiratoria aguda causada por este virus, que filogenéticamente está estrechamente relacionado con SARS-CoV" (Castro, 2020, 1).

Por lo que "la contingencia derivada de la pandemia covid-19 ha puesto contra la pared a gobiernos, sistemas de salud, a la economía global y a las instituciones educativas" (Sanz, 2020, como se citó en 
Sánchez et al., 2020, p. 17), esta enfermedad ha causado graves impacto en muchos ámbitos pero "en la educación superior uno de los efectos inmediatos más importantes es el súbito golpe de timón para migrar totalmente a la modalidad de educación a distancia, a través de educación mediada por tecnología" (The Chronicle of Higher Education, 2020, como se citó en Sánchez et al., 2020, p. 17), obligando a las instituciones de salud a seguir medidas preventivas contra esta situación, motivo por el cual la educación de tipo presencial ha migrado a una educación a distancia por medio de plataformas electrónicas, dejando a un lado los salones de clases e induciendo a la educación en línea.

La decisión de "la suspensión obligatoria de las clases en todos los niveles educativos, con el confinamiento en casa de estudiantes y profesores, ha creado una red de efectos múltiples en todos los actores del proceso educativo" (Mejía et al., 2020, 1384) siendo la motivación académica un rubro afectado.

Los estudiantes de posgrado de la Universidad Autónoma del Noreste principalmente del grado de maestría ante esta nueva situación derivada de la contingencia sanitaria han tenido que lidiar en su vida no sólo con problemas de salud sino también emocionales, económicos y laborales. Algunos problemas de características personales como "la falta de actitud de logro en el crecimiento profesional, la incompatibilidad del tiempo dedicado al trabajo y a los estudios, el poco interés por los estudios, por la carrera y por la institución en la que estudia" (Gil et al., 2019, 27) aunado a que en el ámbito académico se han visto en la necesidad de adecuarse a la migración de sesiones presenciales a sesiones a distancia para continuar con su formación. Por lo que este conjunto de problemas nos hace dudar acerca del grado de motivación académica de los estudiantes durante esta pandemia, el cual puede llegar al grado de "como en muchos casos en la deserción estudiantil universitaria" (Gil et al., 2019, 27).

El interés de este trabajo de investigación se centra en la relación de los factores de regulación externa con la motivación de los estudiantes de maestría en el escenario de clases virtuales y a distancia por emergencia sanitaria y tiene por objetivo verificar de manera estadística la existencia de relación entre la motivación por adquirir nuevos conocimientos y los factores de regulación externa del alumno de maestrías en salud ocupacional y terapia familiar de la Universidad Autónoma del Noreste campus Monclova durante emergencia sanitaria por SARS-CoV2, para ello se formula la hipótesis alternativa "existe relación significativa entre los factores de regulación externa y la motivación para adquirir conocimientos por parte de los alumnos de 
las maestrías de salud ocupacional y terapia familiar de la Universidad Autónoma del Noreste campus Monclova durante emergencia sanitaria por SARS-CoV2" y la hipótesis nula "no existe relación significativa entre los factores de regulación externa y la motivación para adquirir conocimientos por parte de los alumnos de las maestrías de salud ocupacional y terapia familiar de la Universidad Autónoma del Noreste campus Monclova durante emergencia sanitaria por SARS-CoV2”.

Para el contraste de la hipótesis se utilizará pruebas estadísticas de correlación de Spearman para observar relación significativa entre los factores de regulación externa y la motivación para adquirir conocimientos de los alumnos.

\section{MARCO TEÓRICO}

El autor Núñez (2009) nos muestra una definición clásica de motivación sería "un conjunto de procesos implicados en la activación, dirección y persistencia de la conducta" (p. 43), como también "determina la dirección, intensidad y el sentido del comportamiento humano" (Romero et al, 2019, 57), por lo que para Bruno et al. (2020).la motivación "alude a las distintas razones que guían la conducta humana" ( $p$. 131). Por lo que "la motivación es el motor de la conducta humana" (Hernández y Romero, 2020, p. 4).

$\mathrm{Al}$ ser la motivación "un factor de primer orden para afrontar un aprendizaje más autónomo con mayor nivel de exigencia y esfuerzo en el estudiante" (Romero y Pérez, 2009, como se citó en Pegalajar, 2020, 258). Es por ello que "el estudio de la motivación ha despertado especial interés en el ámbito académico en virtud de su vinculación con los desempeños estudiantiles en diferentes estratos del sistema educativo" (Bruno et al., 2020, p. 131).

En el ámbito académico "la motivación es un factor esencial para la persecución de las metas, lo cual permite que los aprendices sean más receptivos y eficaces” (Hernández y Romero, 2020, p. 2).

La motivación académica puede ser conceptualizada como "un proceso cognitivo que incide sobre el papel de los pensamientos del estudiante, sus creencias y emociones y está dirigido hacia un objetivo o meta que la provoca y mantiene (Pintrich y Schunk, 2006 como se citó en Pegalajar, 2020, 258).siendo la motivación académica la que según Herrera et al. (2004) como se citó en Cahuana et al. (2020, p. 3) es "un proceso que activa, direcciona, y sobre todo persevera en una conducta para alcanzar un determinado objetivo", y es en este proceso donde según Zimmerman y Martínez-Pons (1992) como se citó en Pintrich \& Schunk, 2006; Gil et al. (2019, p. 28) el estudiante “dirige la acción de aprender, mostrándose comprometido con toda acción que estime importante para ayudarlo a adquirir conocimientos, desde 
atender con detenimiento las enseñanzas del profesor, hasta ordenar y organizar el material educativo correspondiente, entre otras tareas", debido a que "establece uno de los componentes psicoeducativos que más influencia tiene en el aprendizaje" (Hernández y Romero, 2020, p. 2).

La motivación puede ser dividida de acuerdo a la fuente, siendo "intrínseca cuando nace de la propia persona y su relación con la actividad y es extrínseca cuando los factores del exterior impulsan al individuo a la realización de una actividad establecida" (Hernández y Romero, 2020, p. 4). Los factores de regulación externa son aquellos en los cuales "el propósito deja de estar orientado a la satisfacción personal para buscar el reconocimiento exterior a través de premios y elogios" (González y Rodríguez, 2017, p. 135).

En cuanto a la enseñanza a distancia es definida como un "sistema tecnológico de comunicación bidireccional (multidireccional), que puede ser masivo, basado en la acción sistemática y conjunta de recursos didácticos, y el apoyo de una organización y tutoría que, separados físicamente de los estudiantes, propician en éstos un aprendizaje independiente" (Gil, 2002, p. 89).

\section{MÉTODO}

Para la presente investigación se realizó un "análisis documental de la bibliografía recopilada para el tratamiento del marco teórico y el análisis y comparación en el procesamiento de la información obtenida" como lo sugiere (Hernández et al., 2015, p. 460). Además, es un estudio descriptivo, los cuales "miden, evalúan o recolectan datos sobre diversos conceptos (variables), aspectos, dimensiones o componentes del fenómeno a investigar" (Hernández et al., 1998, p. 102) que busca “conocer la relación que existe entre dos o más conceptos, categorías o variables en un contexto en particular" por lo que es correlacional de acuerdo a lo descrito por Hernández et al (1998, pp.102 y 105).

La investigación se llevó se realizó en la Universidad Autónoma del Noreste campus Monclova teniendo como población objeto de estudio el grupo específico de alumnos de posgrado de las maestrías de Salud Ocupacional y Terapia Familiar, los cuales están conformados por hombres y mujeres con una antigüedad en sus estudios de grado de maestría que va desde pocos meses hasta 2 años.

Para efectuar el cálculo de la muestra se utilizó la calculadora en línea Netquest, que es una calculadora estadística para obtener el tamaño de una muestra. El universo fue de 48 personas, siendo el nivel de confianza considerado del $95 \%$ y un margen de error del $5 \%$. La interpretación de los resultados del cálculo puede leerse de la siguiente manera: "si encuestas a 35 personas, $95 \%$ de las veces el dato real que 
buscas estará en el intervalo, y un margen de error de $\pm 5 \%$ de las veces respecto al dato que observas en la encuesta" (Netquest).

El trabajo de campo se realizó de febrero a marzo de 2021, tiempo durante el cual se realizaron las encuestas vía electrónica, de manera aleatoria a "una muestra no probabilística o dirigida, en la que se seleccionaron sujetos "típicos", con la vaga esperanza de que fueran casos representativos de una población determinada" (Hernández et al., 1998, p. 262). Se utilizó el instrumento de medición "Escala de Motivación Educativa" (Núñez et al., 2005) sin modificación de los elementos, para facilitar su aplicación por las condiciones no presenciales de los grupos en la universidad se envió vía electrónica a las personas que conforman el universo de estudio.

Las variables consideradas son la motivación por adquirir conocimiento y los factores de regulación externa, mismas que conforman el instrumento de medición y se determinaron con base en lo propuesto por Vara (2010, p. 274) para quien "las variables intervinientes pueden ser identificadas mediante la revisión bibliográfica". Y para este caso son variables nominales con siete categorías, siendo éstas politómicas con diversas opciones de respuesta (opción 1, no corresponde en lo absoluto, opción 2, se corresponde muy poco, opción 3, corresponde poco, opción 4 corresponde medianamente, opción 5, corresponde bastante, opción 6 corresponde mucho, opción 7, corresponde totalmente).

Los elementos del instrumento de medición que tomaremos como correspondientes a las variables "conocimientos" y "regulación externa" son descritos en la tabla 1:

Tabla 1 Variables.

$\begin{array}{ll}\text { MConocimiento1 } & \begin{array}{l}\text { Porque para mí es un placer y una } \\ \text { satisfacción aprender cosas nuevas. }\end{array} \\ \text { MConocimiento2 } & \begin{array}{l}\text { Por el placer de descubrir cosas nuevas } \\ \text { desconocidas para mí. }\end{array} \\ \text { MConocimiento3 } & \begin{array}{l}\text { Por el placer de saber más sobre las } \\ \text { asignaturas que me atraen. }\end{array} \\ \text { MConocimiento4 } & \begin{array}{l}\text { Porque mis estudios me permiten } \\ \text { continuar aprendiendo un montón de } \\ \text { cosas que me interesan. }\end{array} \\ \text { RegulaciónExterna1 } & \begin{array}{l}\text { Porque sólo con el Bachillerato no } \\ \text { podría encontrar un empleo bien } \\ \text { pagado. } \\ \text { Para poder conseguir en el futuro un } \\ \text { trabajo más prestigioso. }\end{array} \\ \text { RegulaciónExterna2 } & \end{array}$




\section{RegulaciónExterna3 Porque en el futuro quiero tener una "buena vida". \\ RegulaciónExterna4 \\ Para tener un sueldo mejor en el futuro.}

Fuente: Elaboración propia.

\section{RESULTADOS}

Al aplicar la correlación de Spearman mediante el software estadístico SPSS en los datos, los valores de la significancia bilateral para la correlación entre las variables "RegulacionExterna1" y "MConocimiento1" es de 0.456 , para la correlación entre las variables "RegulacionExterna1" y "MConocimiento2" es de 0.224 , mientras que para la correlación entre variables "RegulacionExterna1" y "MConocimiento3" es de 0.268 y para las variables "RegulacionExterna1 y "MConocimiento4" es de 0.710. Los valores de la significancia bilateral para las correlaciones entre las variables "RegulacionExterna2" y "MConocimiento1" es de 0.824 , para las variables "RegulacionExterna2" y "MConocimiento2" es de 0.933 , mientras que para las variables "RegulacionExterna2" y "MConocimiento3" es de 0.774 y para "RegulacionExterna2" y "MConocimiento4" el valor es de 0.558 como lo podemos observar en la figura 1:

Figura 1. Correlación de variables "conocimientos" y "regulación externa"

\begin{tabular}{|c|c|c|c|c|}
\hline \multicolumn{5}{|c|}{ Correlaciones } \\
\hline & & & $\begin{array}{c}\text { RegulacionExt } \\
\text { ema1 }\end{array}$ & $\begin{array}{c}\text { RegulacionExt } \\
\text { ema2 }\end{array}$ \\
\hline \multirow[t]{12}{*}{ Rho de Spearman } & \multirow[t]{3}{*}{ MiConocimiento 1} & Coeficiente de comelación & -.130 & 039 \\
\hline & & Sig. (bilateral) & .456 & .824 \\
\hline & & $N$ & 35 & 35 \\
\hline & \multirow[t]{3}{*}{ MConocimiento2 } & Coeficiente de comelación & -.211 & 015 \\
\hline & & Sig. (bilateral) & .224 & .933 \\
\hline & & $N$ & 35 & 35 \\
\hline & \multirow[t]{3}{*}{ MiConocimiento 3} & Coeficiente de comelación &,- 193 &, 050 \\
\hline & & Sig. (bilateral) & .268 & .774 \\
\hline & & $\mathrm{N}$ & 35 & 35 \\
\hline & \multirow[t]{3}{*}{ MiConocimiento 4} & Coeficiente de comelación & 065 & .102 \\
\hline & & Sig. (bilateral) & .710 & .558 \\
\hline & & $N$ & 35 & 35 \\
\hline
\end{tabular}

Fuente: Elaboración propia mediante software estadístico SPSS. 
Al aplicar la correlación de Spearman mediante el software estadístico SPSS en los datos, los valores de la significancia bilateral para la correlación entre las variables "RegulacionExterna3" y "MConocimiento1" es de 0.202, para la correlación entre las variables "RegulacionExterna3" y “MConocimiento2" es de 0.415 , mientras que para la correlación entre variables "RegulacionExterna3" y "MConocimiento3" es de 0.299 y para las variables "RegulacionExterna3 y "MConocimiento4" es de 0..029. Los valores de la significancia bilateral para las correlaciones entre las variables "RegulacionExterna4" y "MConocimiento1" es de 0.516, para las variables "RegulacionExterna4" y "MConocimiento2" es de 0.330 , mientras que para las variables "RegulacionExterna4" y "MConocimiento3" es de 0.338 y para "RegulacionExterna4" y "MConocimiento4" el valor es de 0.649 como lo podemos observar en la figura 2 :

Figura 2. Correlación de variables "conocimientos" y "regulación externa"

\begin{tabular}{|c|c|c|c|c|}
\hline \multicolumn{5}{|c|}{ Correlaciones } \\
\hline & & & $\begin{array}{l}\text { RegulacionExt } \\
\text { ema3 }\end{array}$ & $\begin{array}{l}\text { Regulacion Ext } \\
\text { ema } 4\end{array}$ \\
\hline \multirow[t]{12}{*}{ Rho de Spearman } & \multirow[t]{3}{*}{ MConocimiento 1} & Coeficiente de correlación & .221 & -.113 \\
\hline & & Sig. (bilateral) & .202 & .516 \\
\hline & & $\mathrm{N}$ & 35 & 35 \\
\hline & \multirow[t]{3}{*}{ MiConocimiento2 } & Coeficiente de comelación & .142 & -.170 \\
\hline & & Sig. (bilateral) & .415 & .330 \\
\hline & & $\mathrm{N}$ & 35 & 35 \\
\hline & \multirow[t]{3}{*}{ MiConocimiento 3} & Coeficiente de correlación & .181 & -.167 \\
\hline & & Sig. (bilateral) & .299 & .338 \\
\hline & & $N$ & 35 & 35 \\
\hline & \multirow[t]{3}{*}{ MiConocimiento 4} & Coeficiente de correlación & $.369^{\circ}$ & 080 \\
\hline & & Sig. (bilateral) & .029 & .649 \\
\hline & & $N$ & 35 & 35 \\
\hline
\end{tabular}

Fuente: Elaboración propia mediante software estadístico SPSS.

Los valores de la significancia bilateral superior a 0.05 muestran que durante la emergencia sanitaria por SARS-CoV2 los alumnos de posgrado de las maestrías de salud ocupacional y terapia familiar de la
Universidad Autónoma del Noreste campus Monclova muestran una correlación entre factores de motivación intrínsecos para adquirir conocimiento con factores de motivación extrínsecos. 
Los alumnos están motivados para

Factor extrínseco: “Con el Bachillerato no podría encontrar un empleo bien pagado".

Los alumnos están motivados para adquirir conocimiento debido a que es un placer y una satisfacción aprender cosas nuevas porque sólo con el Bachillerato no podría encontrar un empleo bien pagado.

Los alumnos están motivados para adquirir conocimiento por el placer de descubrir cosas nuevas desconocidas debido a que sólo con el Bachillerato no podría encontrar un empleo bien pagado.

Los alumnos están motivados para adquirir conocimiento por el placer de saber más sobre las asignaturas que me atraen debido a que sólo con el Bachillerato no podría encontrar un empleo bien pagado.

Los alumnos están motivados para adquirir conocimiento porque mis estudios me permiten continuar aprendiendo un montón de cosas que me interesan debido a que sólo con el Bachillerato no podría encontrar un empleo bien pagado.

Factor extrínseco: "Para poder conseguir en el futuro un trabajo más prestigioso".

Los alumnos están motivados para adquirir conocimiento debido a que es un placer y una satisfacción aprender cosas nuevas para poder conseguir en el futuro un trabajo más prestigioso. adquirir conocimiento por el placer de descubrir cosas nuevas desconocidas para poder conseguir en el futuro un trabajo más prestigioso.

Los alumnos están motivados para adquirir conocimiento por el placer de saber más sobre las asignaturas para poder conseguir en el futuro un trabajo más prestigioso.

Los alumnos están motivados para adquirir conocimiento porque los estudios les permiten continuar aprendiendo un montón de cosas que me interesan para poder conseguir en el futuro un trabajo más prestigioso.

Factor extrínseco: "Porque en el futuro quiero tener una "buena vida".

Los alumnos están motivados para adquirir conocimiento debido a que es un placer y una satisfacción aprender cosas nuevas porque en el futuro quiero tener una "buena vida".

Los alumnos están motivados para adquirir conocimiento por el placer de descubrir cosas nuevas desconocidas porque en el futuro quiero tener una "buena vida".

Los alumnos están motivados para adquirir conocimiento por el placer de saber más sobre las asignaturas que me atraen porque en el futuro quiero tener una "buena vida". 
Factor extrínseco: "Para tener un sueldo mejor en el futuro".

Los alumnos están motivados para adquirir conocimiento debido a que es un placer y una satisfacción aprender cosas nuevas para tener un sueldo mejor en el futuro.

Los alumnos están motivados para adquirir conocimiento por el placer de descubrir cosas nuevas desconocidas para tener un sueldo mejor en el futuro.

Los alumnos están motivados para adquirir conocimiento por el placer de saber más sobre las asignaturas para tener un sueldo mejor en el futuro.

Los alumnos están motivados para adquirir conocimiento porque los estudios les permiten continuar aprendiendo un montón de cosas que les interesan para tener un sueldo mejor en el futuro.

Los valores de la significancia bilateral inferior a 0.05 muestran que durante la emergencia sanitaria por SARS-CoV2 los alumnos de posgrado de las maestrías de salud ocupacional y terapia familiar de la Universidad Autónoma del Noreste campus Monclova no muestran una correlación entre factores de motivación intrínsecos para adquirir conocimiento con factores de motivación extrínsecos en la relación "los alumnos están motivados para adquirir conocimiento porque los estudios les permiten continuar aprendiendo un montón de cosas que me interesan porque en el futuro quiero tener una buena vida".

Al no ser el valor de la significancia bilateral superior a 0.05 en todas las correlaciones entre variables de motivación por adquirir conocimientos y factores de regulación externos se procede a rechazar la hipótesis alternativa y no rechazar la hipótesis nula "no existe relación significativa entre los factores de regulación externa y la motivación para adquirir conocimientos por parte de los alumnos de las maestrías de salud ocupacional y terapia familiar de la Universidad Autónoma del Noreste campus Monclova durante emergencia sanitaria por SARS-CoV2”.

\section{CONCLUSIONES}

Los valores de la significancia bilateral superior a 0.05 muestran que durante la emergencia sanitaria por SARS-CoV2 los alumnos de posgrado de las maestrías de salud ocupacional y terapia familiar de la Universidad Autónoma del Noreste campus Monclova están motivados para adquirir conocimiento debido a que es un placer y una satisfacción aprender cosas nuevas, descubrir cosas desconocidas sobre las asignaturas que les atraen y porque sus estudios les permiten continuar aprendiendo un montón de cosas que les interesan debido a que perciben que sólo con el Bachillerato no podría encontrar un empleo bien pagado, y/o en el futuro un trabajo más prestigioso y/o para tener un sueldo mejor en el futuro. 
Así mismo los alumnos de posgrado de las maestrías de salud ocupacional y terapia familiar de la Universidad Autónoma del Noreste campus Monclova están motivados para adquirir conocimiento debido a que es un placer y una satisfacción aprender cosas nuevas, descubrir cosas desconocidas porque sus estudios les permiten continuar aprendiendo un montón de cosas que les interesan debido a que perciben que esto les ayudará a tener una buena vida pero no toman en cuenta que los estudios les permiten continuar aprendiendo un montón de cosas que me interesan para esto.
Al no ser el valor de la significancia bilateral superior a 0.05 en todas las correlaciones entre variables de motivación por adquirir conocimientos y factores de regulación externos se procede a rechazar la hipótesis alternativa y no rechazar la hipótesis nula "no existe relación significativa entre los factores de regulación externa y la motivación para adquirir conocimientos por parte de los alumnos de las maestrías de salud ocupacional y terapia familiar de la Universidad Autónoma del Noreste campus Monclova durante emergencia sanitaria por SARS-CoV2". 


\section{REFERENCIAS}

Álvarez, N. A., Osiadacz, F. I., Paci, M. J., Pereira, M. T., Pérez, F. J., Soto, V. I., \& Zorzano, B. M. (2020). Efectos de la pandemia COVID-19 en la práctica deportiva de jugadoras de hockey sobre césped de la categoría sub-14 y sub-16 del Club Deportivo Universidad Católica y Prince of Wales Country Club (Doctoral dissertation, Universidad Andrés Bello).En http://repositorio.unab.cl/xmlui/bitstream/handle/ria/18599/a131582_Alvarez_N_Efecto_de _la_pandemia_2020.pdf?sequence=1\&isAllowed=y

Balletti, R. P. (2020). Enfermedades epidémicas y pandémicas: Causas, cronología e implicaciones socioculturales. Anales de la Real Academia de Farmacia, 86(3), 189-213. En https://analesranf.com/wp-content/uploads/2020/86_03/8603_04.pdf

Bruno, F. E., Liporace, M. F., \& Stover, J. B. (2020). Escala de motivación situacional académica para estudiantes universitarios: Desarrollo y análisis psicométricos. Interdisciplinaria. Revista de Psicología y Ciencias Afines, 37(1), 1-29.

Cahuana, M, Mamani-Benito, O., \& Carranza R. F. (2020). Autoconcepto académico y motivación académica en jóvenes talento del departamento de Puno, Perú. Propósitos y

Representaciones, 8(3), 788. https://dx.doi.org/10.20511/pyr2020.v8n3.788

Castro L., Ricardo. (2020). Coronavirus, una historia en desarrollo. Revista médica de Chile, 148(2), 143-144. https://dx.doi.org/10.4067/s0034-98872020000200143

Galli, C. M. (2020). La vida y la muerte en la primera pandemia global. https://repositorio.uca.edu.ar/bitstream/123456789/10623/1/vida-muerte-primerapandemia.pdf

Gil Rivera, María del Carmen. (2000). Educación a distancia: De la teoría a la práctica. Perfiles educativos, 22(88), 89-92.

Gil, J. M., Fuster, F. G., Norabuena, R. P., Maldonado, H. W. Norabuena, E. D., \& Hernández, R. M. (2019). Motivación académica y su influencia en el desarrollo de las capacidades de estudiantes en el área de inglés. Revista de Psicología, 15(30), 26-41.

González-Peiteado, M., \& Rodríguez-López, B. (2017). Factores motivacionales de los adultos para el estudio de una lengua extranjera. Pedagogía Social. Revista Interuniversitaria, (30), 129143.

Hernández, C., Garcés, M. F., \& Hernández, E. (2020). COVID-19: LOS PRIMEROS 40 DÍAS DE UNA PANDEMIA. Acta Científica, 23(1), 58-100. En

Hernández, J. V., \& Romero, G. H. (2020). Influencia del Liderazgo docente en la motivación de universitarios. IJERI: International Journal of Educational Research and Innovation, (13), $1-16$.

Hernández, R., Fernández, C., y Baptista, P. (1998). Metodología de la investigación.:McGraw Hill.

Hernández, R., Fernández, C., y Baptista, P. (2015). Metodología de la investigación. McGraw Hill. https://www.svbe.org/descargas/Acta\%20Cient\%C3\%ADfica\%202020-1.pdf\#page=62.

Mejía, O. G, Casquete, N. E, \& Castro, C. R. M. (2020). La educación y el aprendizaje ante el Covid-19. Dominio de las Ciencias, 6(3), 1382-1400. En file:///C:/Documents/HISTORICO\%20MODULO\%204/Downloads/DialnetLaEducacionYElAprendizajeAnteElCovid19-7562479.pdf

Muñiz, C., \& Corduneanu, V. I. (2020). Percepción de riesgo y consumo mediático durante el inicio de la pandemia de COVID-19 en México. Más poder local, (41), 44-47.

Núñez, J. C. (2009, September). Motivación, aprendizaje y rendimiento académico. In Trabajo presentado en el X Congresso Internacional Galego-Português de Psicopedagogia. Braga, Portugal (pp. 41-67).

Paniagua, E. (2020). VIVENCIA DE FAMILIARES DE PERSONAS AFECTADAS POR COVID-19. Academic Disclosure, 1(1), 153-163.

Pegalajar-Palomino, M. C. (2020). Relación entre la motivación académico-personal del estudiante novel en educación y las estrategias de trabajo autónomo. Formación universitaria, 13(5), 257-268. 
Romero Pileta, I., Dopico Pérez, H. M., Fernández Téllez, I., Montoro Bombú, R., Chávez Cevallos, E., \& Contreras Calle, W. T. (2019). Análisis integral de la motivación en boxeadores. Revista Cubana de Investigaciones Biomédicas, 38(2), 56-72.

Sánchez, M., Martínez, A. M., Torres, R., Agüero, M., Hernández, A. K., Benavidez, M. A., Rendón, V. J., Jaimes, C. A. (2020). Retos educativos durante la pandemia de COVID-19: una encuesta a profesores de la UNAM. Revista Digital Universitaria, 21(3), 1-24. En https://www.revista.unam.mx/wp-content/uploads/a12.pdf

Vara, A. A. (2010). Desde La Idea hasta la sustentación: Siete pasos para una tesis exitosa. Un método efectivo para las ciencias empresariales. Lima: Instituto de Investigación de la Facultad de Ciencias Administrativas y Recursos Humanos. Universidad de San Martín de Porres. 